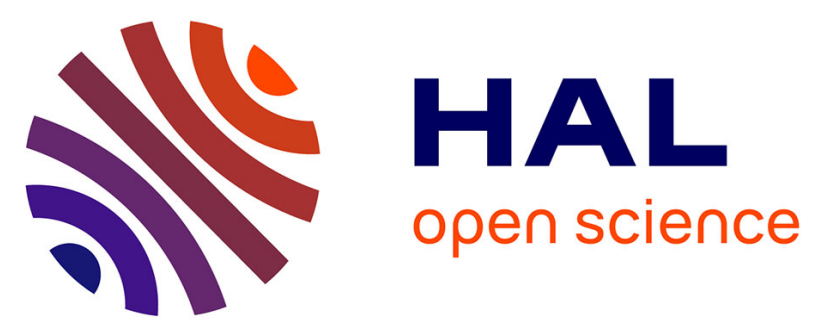

\title{
Thermodynamics study of the noncovalent functionalization of surfactant suspended graphene nanosheets with porphyrin molecules
}

Géraud Delport, Fabien Vialla, Stéphane Campidelli, Christophe Voisin, Jean-sébastien Lauret

\section{To cite this version:}

Géraud Delport, Fabien Vialla, Stéphane Campidelli, Christophe Voisin, Jean-sébastien Lauret. Thermodynamics study of the noncovalent functionalization of surfactant suspended graphene nanosheets with porphyrin molecules. physica status solidi (b), 2016, 253, pp.2373-2376. 10.1002/pssb.201600269 . cea-01414729

HAL Id: cea-01414729

https://hal-cea.archives-ouvertes.fr/cea-01414729

Submitted on 8 Oct 2021

HAL is a multi-disciplinary open access archive for the deposit and dissemination of scientific research documents, whether they are published or not. The documents may come from teaching and research institutions in France or abroad, or from public or private research centers.
L'archive ouverte pluridisciplinaire HAL, est destinée au dépôt et à la diffusion de documents scientifiques de niveau recherche, publiés ou non, émanant des établissements d'enseignement et de recherche français ou étrangers, des laboratoires publics ou privés. 


\title{
Thermodynamics study of the noncovalent functionalization of surfactant suspended graphene nanosheets with porphyrin molecules.
}

\author{
Géraud Delport ${ }^{1}$, Fabien Vialla², Stéphane Campidelli ${ }^{3}$, Christophe Voisin ${ }^{2}$, Jean Sébastien Lauret ${ }^{*}, 1$ \\ ${ }^{1}$ Laboratoire Aimé Cotton, CNRS, Univ. Paris-Sud, ENS Cachan, Université Paris-Saclay, 91405 Orsay Cedex, France \\ ${ }^{2}$ Laboratoire Pierre Aigrain, Ecole Normale Supérieure, CNRS, UPMC, Université Paris Diderot, Paris, France \\ ${ }^{3}$ LICSEN, NIMBE, CEA, CNRS, Université Paris-Saclay, CEA Saclay 91191 Gif-sur-Yvette Cedex, France
}

Received XXXX, revised XXXX, accepted XXXX

Published online XXXX

Key words: nanomaterials, noncovalent functionalization, graphene, energy transfer, photoluminescence.

* Corresponding author: e-mail jean-sebastien.lauret@lac.u-psud.fr, Phone: +33-1-69352132

We report on the noncovalent functionalization of graphene with hydrophobic tetraphenyl porphyrin (TPP) molecules in micellar aqueous suspensions. We study the thermodynamical parameters of the reaction using optical spectroscopy, and measure its Gibbs energy. We show that a total reaction can be achieved, leading to a high functionalization yield, despite the $\pi$ - stacking noncovalent binding.

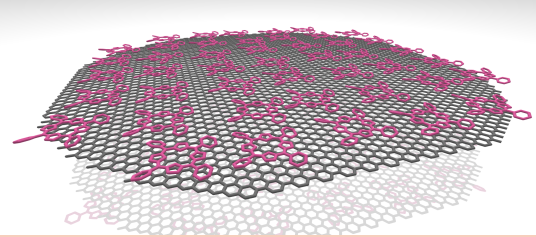

Side view of a graphene nanosheet covered with porphyrins.
1 Introduction Functionalizing nanomaterials has been a subject of intensive research for decades [1-3]. Coupling carbon materials with organic molecules offers unique possibilities to bring new functionalities, benefiting from the wide tunability of the properties of organic molecules, such as energy transfer [4,5], surface protection [6] and enhanced light emission [7]. In this context, nanocompounds with charge or energy transfer properties are of great interest, especially for optoelectronics [8] and biology [9].

Nonradiative energy transfer from $\pi$-stacked hydrophobic porphyrin molecules to single wall carbon nanotubes (SWCNT) [10-14] and graphene [15,16] was demonstrated a few years ago. Such hybrid nano compounds are designed through noncovalent functionalization techniques, offering the advantage of preserving the $s p^{2}$ carbon structure to maintain good optical and elec- tronic properties. However, such noncovalent reactions are likely to produce poor reproducibility and low stability over time. Herein, we report on an easy and reproducible route to synthesize stable graphene/porphyrin complexes in micelles environment, exhibiting energy transfer properties. We perform a thermodynamical study to extract the Gibbs energy of this reaction, using a Hill adsorption model. Finally, we compare this $\pi$-stacking effect on graphene with previous results reported on SWCNTs [17] and DFT calculations [18].

2 Chemical method Reaction (1) is performed simply by letting evolve a suspension obtained by mixing surfactant suspensions of TPP and graphene sheets, in a $50 \%-50 \%$ volume proportion. In the following, we monitor the functionalization process by a combination of optical absorption spectroscopy (OAS) and photoluminescence 


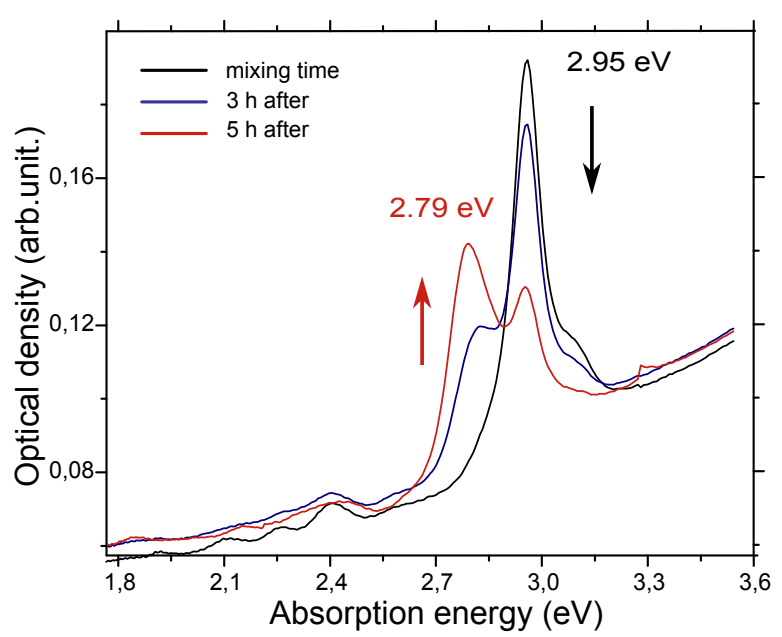

Figure 1 Absorption spectra of the sample for a TPP concentration of $8 \mu \mathrm{mol} . \mathrm{L}^{-1}$ (arrows symbolise evolution over time)

(PL). This unassisted reaction route allows a progressive optical study of the reaction that would be more difficult if sonication or stirring were involved.

$$
\mathrm{TPP}_{s c}+\mathrm{gr}_{s c}=\mathrm{TPP} / \mathrm{gr}_{s c}
$$

The graphene material is purchased from NanoIntegris (PureSheet MONO[19]). The sample contains one to few layers graphene flakes in the proportion written below in Tab.(1). The graphene concentration is $0.05 \mathrm{mg} . \mathrm{mL}^{-1}$, with an average flake area of $10^{4} \mathrm{~nm}^{2}$ and a large statistical dispersion in size [19]. The graphene sheets are dispersed in a $2 \mathrm{wt} \%$ sodium cholate (SC) solution [20].

Table 1 Proportions of layers among the graphene sheets

\begin{tabular}{l|llll}
\hline & 1 layer & 2-layers & 3-layers & 4-layers \\
\hline Proportion (\%) & 6 & 23 & 27 & 5 \\
\hline
\end{tabular}

In order to obtain isolated the TPP suspension, we used the micelle swelling method $[21,14]$ to efficiently transfer TPP molecules from their dichloromethane solvent to a $2 \mathrm{wt} \% \mathrm{SC}$ suspension in a $\mathrm{pH} 8$ borate buffer. After three hours of soft tip sonication, the TPP cholate suspension is centrifugated at $6300 \mathrm{~g}$ for 10 minutes, in order to remove molecular aggregates and remaining dichloromethane. To study the chemical equilibrium, we realized several samples with a fixed graphene concentration of $0.025 \mathrm{mg} \cdot \mathrm{mL}^{-1}$ and various TPP concentrations between and 0 and $50 \mu \mathrm{mol} . \mathrm{L}^{-1}$.

3 Absorption monitoring of the reaction Fig.1a) displays the optical density of a sample including $8 \mu \mathrm{mol}^{-\mathrm{L}^{-1}}$ of TPP at several stages of the reaction. At the initial time, the absorption spectrum solely displays the free porphyrins response, with its main Soret band peak at $2.95 \mathrm{eV}$ [22]. After a few minutes, an additional resonance grows sponteaneously at $2.79 \mathrm{eV}$ and reaches saturation within five hours. It should be noted that the spectra show an isobestic point, indicative of the conversion of one chemical species into another [17]. In all samples, the final absorption spectrum shows an equilibrium, with both shifted and unshifted populations. The shifted Soret band at $2.79 \mathrm{eV}$ can be attributed to TPP stacked on graphene [14,23], whereas the one at $2.95 \mathrm{eV}$ reflects the presence of free TPP in the suspension.

$4 \mathrm{PL}$ quenching and energy transfer We performed PL experiments to evaluate the influence of graphene on the luminescence efficiency of the chromophores. We monitored the Q bands emission [22] signal between $2.06 \mathrm{eV}$ and $1.65 \mathrm{eV}$, for samples with and without graphene nanosheets.

Fig.2 a) represents the photoluminescence signal of TPP samples, for a monochromatic light excitation at 2.95 $\mathrm{eV}$, for an initial chromophore concentration $[T P P]_{0}$ of $8 \mu \mathrm{mol} . \mathrm{L}^{-1}$. In the presence of graphene sheets, the TPP PL intensity decreases significantly. This quenching of the molecule fluorescence is the signature of an energy transfer from the TPP to the graphene sheets. The efficiency of the energy transfer from TPP molecules to the graphene sheets can be written as :

$$
\eta=1-\frac{I_{T P P / g r}}{I_{T P P_{0}}}
$$

with $I_{T P P / g r}$ and $I_{T P P_{0}}$ the emission intensity of the TPP in presence and in absence of graphene respectively.

On Fig. 2 a), the red curves corresponds to the PL signal $I_{T P P_{0}}$ of the reference TPP solution. The black curve displays the PL measured on the graphene/porphyrin suspension $\left(I_{t o t}\right)$. As stated in section 3, the suspension contains both free and $\pi$-stacked porphyrins. Therefore, this PL signal $\left(I_{t o t}\right)$ is the sum of the contributions from the free porphyrin $\left(\mathrm{I}_{T P P_{e q}}\right)$ and the porphyrin stacked on graphene $\left(\mathrm{I}_{T P P / g r}\right): I_{t o t}=I_{T P P_{e q}}+I_{T P P / g r}$. In order to evaluate the transfer efficiency, we need to know the value of $\mathbf{I}_{T P P / g r}$. From the absorption spectrum at the equilibrium reported on Fig. 1, the final free TPP concentration can be estimated to be $30 \pm 5 \%$ of the reference porphyrin solution leading to $I_{T P P_{e q}} \simeq 0.3 \times I_{T P P_{0}}$. From the formula $I_{T P P / g r}=I_{t o t}-0.3 \times I_{T P P_{0}}$, we estimate $I_{T P P / g r}$ to be close to $1 \%$ of $I_{T P P_{0}}$. Hence, the remaining TPP photoluminescence signal essentially comes from the free molecules. Then, we can estimate that $\eta=99 \pm 5 \%$. This near unity efficiency is comparable to the value found by $\mathrm{Xu}$ et al. for ionic hybrid compounds [15] and for nanotube/TPP samples for which a transfer efficiency of $1-10^{-5}$ was extracted [23]. This is indicative of the presence of TPP molecules at a short distance from the graphene surface. From ab initio calculations, the TPP-graphene distance in our compound was estimated to be $0.3 \mathrm{~nm}$ [18]. At such distance, Malic et al. predicted a transfer efficiency above $99 \%$, using a Förster energy transfer model [5] in good agreement with our findings. 


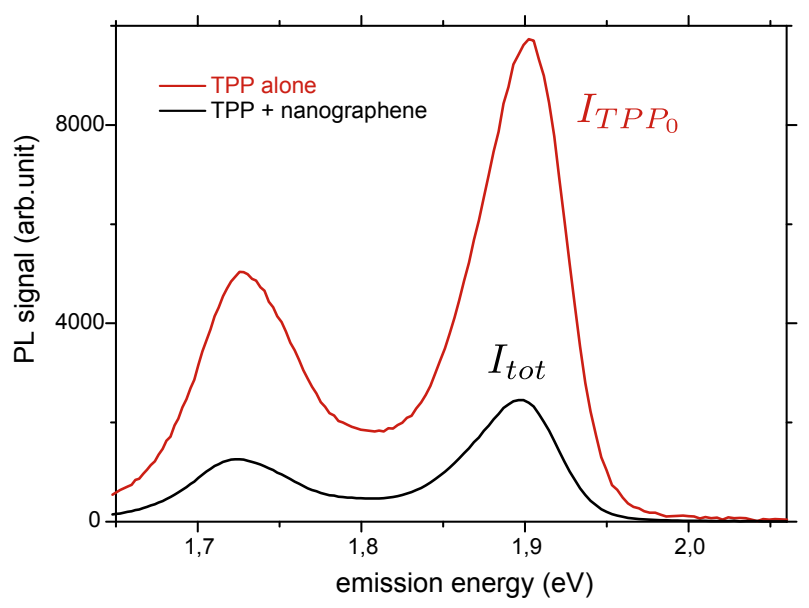

a) Quenching effect of graphene on TPP PL signal for a concentration of $8 \mu \mathrm{mol} . \mathrm{L}^{-1}$

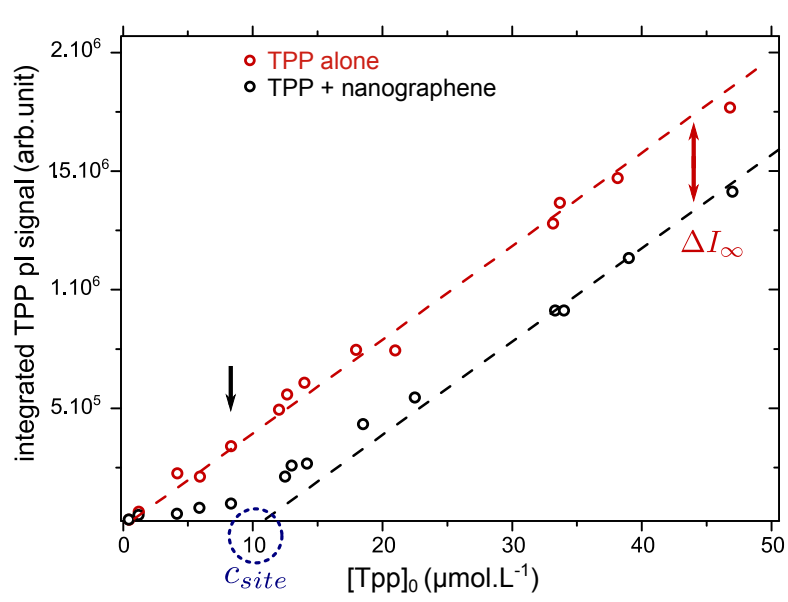

b) PL signal vs concentration with and without graphene sheets (the black arrow highlights the $8 \mu \mathrm{mol} . \mathrm{L}^{-1}$ samples)

Figure 2 Quenching effect of the molecule fluorescence with concentration.

5 thermodynamical study of the stacking reaction We take advantage of these simple reaction conditions to study the stacking equilibrium. Here, we use both the OAS and PL quenching data of TPP to monitor the evolution of the stacking process as we increase the porphyrin initial concentration $[T P P]_{0}$. As shown on Fig. 2 b), the PL signal of TPP molecules in the absence of graphene grows linearly with concentration, indicating that an increasing number of monomers are solubilized. In the presence of graphene, the PL signal also rises with $[T P P]_{0}$ but with a threshold behavior. For $[T P P]_{0}<10 \mu \mathrm{mol} . \mathrm{L}^{-1}$, the PL signal increases with a very moderate slope, smaller than for the lone TPP samples. This indicates that an important fraction of monomers are quenched by their interaction with graphene. However, for concentrations above $10 \mu \mathrm{mol} . \mathrm{L}^{-1}$, the slope of the PL signal eventually recovers the value measured for the free TPP sample. This behavior is typical of a total chemical reaction [17] where the number of $\pi$-stacked TPP saturates once every adsorption site is occupied. Therefore, the concentration of available adsorption sites can be directly quantified from Fig.2 b), as it corresponds to the threshold concentration. We obtain $c_{\text {site }}=10 \pm 2 \mu \mathrm{mol} . \mathrm{L}^{-1}$ through the $\mathrm{x}$-intercept of the linear asymptote for the PL plot in presence of graphene on Fig.2b) [17].

For each TPP concentration, the normalized reaction extent can be written as :

$$
X=\frac{[T P P]_{0}-[T P P]_{e q}}{c_{\text {site }}}=\frac{[T P P / g r]}{c_{\text {site }}}
$$

with $[\mathrm{TPP}]_{0}$ the initial concentration of TPP, $[\mathrm{TPP}]_{e q}$ the concentration at equilibrium and [TPP/gr] the concentration of stacked TPP.
Given the near unity quenching from graphene, the PL intensities are directly proportional to the concentrations of free porphyrin in each samples. Hence, we can express the reaction extent under the following form:

$$
X=\frac{I_{T P P_{0}}-I_{T P P_{e q}}}{\Delta I_{\infty}}
$$

with $\Delta I_{\infty}$ the difference of PL intensity between the two linear curves in Fig.2b) at high concentrations. This $\Delta I_{\infty}$ corresponds to the absence of PL from the total amount of quenched TPP : it is directly proportional to $c_{\text {site }}$.

In Fig.3, we plot this reaction extent $X$ as a function of the free TPP concentration $[T P P]_{e q}$, extracted from the optical density measurement at $2.95 \mathrm{eV}$ through the BeerLambert's Law. This plot yields a saturation curve, characteristic of a Langmuir adsorption isotherm [24]. In order to extract the energetic parameters associated to this equilibrium, we describe the reaction by a cooperative Hill adsorption model [25] :

$$
\mathrm{nTPP}+\mathrm{gr}=\mathrm{TPP}_{n} / \mathrm{gr}
$$

where $n$ stands for the cooperativity of the adsorption reaction. $n>1$ is indicative of a cooperative reaction whereas $n<1$ stands for an anti-cooperative process. The Hill model predicts the evolution of $X$ through a saturation formula [17]:

$$
X=\frac{\left(K[T P P]_{e q}\right)^{n}}{1+\left(K[T P P]_{e q}\right)^{n}}
$$

where $K$ is the thermodynamical equilibrium constant. We obtain $K=1.3 \pm 110^{6}$ and $n=1.3 \pm 0.3$. We extract the free Gibbs energy of the reaction $\Delta r G^{0}=$ $-R T \ln (K) \simeq-35 \mathrm{~kJ} . \mathrm{mol}^{-1}$. These values are of the 


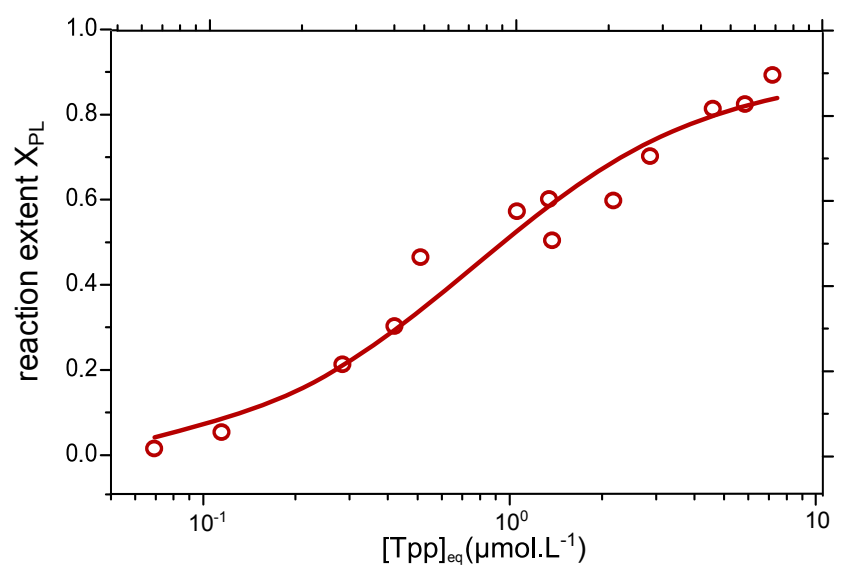

Figure 3 Evolution of the reaction extent in function of the available porphyrin concentration.

same order of magnitude than the one obtained for SWCNTs [17]. We showed in a previous work that the absolute value of the Gibbs energy increases with the SWCNT diameter [17]. This trend was consistent with a recent DFT model from Orellana et al. [18], which found an increase of the adsorption energy of single TPP with nanotube's diameter. For graphene, this DFT model predicted a binding energy twice as high as for the largest HiPCo nanotubes. However, both $n$ and $\Delta \mathrm{rG}^{0}$ measured for graphene are lower than the value obtained for the largest diameter of HiPCo nanotubes: $\langle n\rangle=2.3 \pm 0.8$ and $\Delta \mathrm{rG}^{0} \simeq$ $-42 \mathrm{~kJ} . \mathrm{mol}^{-1}$.

Several reasons could explain the deviation of this model from our measurements on graphene. First of all, the real reaction that we monitor is the exchange between TPP and cholate monomers at the carbon surface. Thus, the Gibbs energy that we evaluate includes other effects than the simple TPP adsorption, such as cholate monomers desorption, or changes in the organization of the global surfactant layer, and these effects may be curvature dependent [26]. Moreover, recent papers studied the interaction between TPP molecules upon adsorption onto a $\mathrm{sp}^{2}$ carbon surface. Pham et al. proved the existence of organized networks of porphyrins stacked on graphene sheets thanks to scanning tunnelling microscopy experiments [27]. Orellana performed additional DFT calcutations [28], showing that TPPs can assemble around SWCNTs into organized rings of 4 to 8 units thanks to porphyrin-porphyrin interactions, leading to a higher adsorption energy on carbon nanotubes. These two papers show that the interaction between porphyrin molecules may play an important role in the adsorption mechanism. Such a cooperative interaction was not included in the model [18] that predicted an increased adsorption energy of TPP on graphene in comparison with nanotubes.

6 Conclusion We have designed a fast and spontaneous route to noncovalently functionalize suspended graphene sheets in solution with TPP molecules. These hybrid compounds show an efficient energy transfer from the molecule to the graphene sheets with an efficiency up to $99 \%$. Moreover, experiments as a function of the TPP concentration allow to gain insight into the thermodynamics of the reaction. The analysis of the reaction extent $X$ with a Hill adsorption model shows that the process is slightly cooperative $(n>1)$ and leads to a Gibbs energy of $-35 \mathrm{~kJ} \mathrm{~mol}^{-1}$. The comparison with SWCNT evidenced that this value of Gibbs energy is not compatible with the prediction of DFT calculations of the variation of the monomer adsorption energy as a function of the curvature of $\mathrm{sp}^{2}$ carbon surfaces. It highlights that more complex processes are involved in the adsorption reaction such as surfactant reorganization or porphyrin-porphyrin interactions.

7 Acknowledgements JSL and CV are members of "Institut Universitaire de France".

\section{References}

[1] M.S. Strano, C.A. Dyke, M.L. Usrey, P.W. Barone, M.J. Allen, H.W. Shan,C Kittrell, R.H. Hauge,J.M. Tour and R.E. Smalley ,Science 301, 1519-1522 (2003) .

[2] M. Zheng, A. Jagota, E.D. Semke, B.A. Diner, R.S. Mclean, S.R. Lustig, R.E. Richardson and N.G. Tassi ,Nat. Mater. 2,338-342 (2003).

[3] V. Georgakilas, M. Otyepka, A.B. Bourlinos, V. Chandra, N. Kim, K.C. Kemp, P. Hobzał, R. Zboril, and K.S. Kim ,Chem. rev. 112, 6156-6214 (2012).

[4] H. Chang,L. Tang, Y.Wang, J. Jiang, and J. Li, Anal.Chem. 82, 2341-2346 (2010)

[5] E. Malic, H. Appel, O.T. Hofmann, and Angel Rubio J.Phys.Chem.C 118, 9283-9289 (2014).

[6] S. Campidelli, B. Ballesteros, A. Filoramo, D.D Diaz, G. de la Torre, T. Torres, G.M.A. Rahman, C. Ehli, D. Kiessling, F. Werner, V. Sgobba, D.M. Guldi, C. Cioffi, M. Prato and J.P. Bourgoin, J.A.C.S. 130, 11503-11509 (2008).

[7] Y. Piao, B. Meany, L.R. Powell, N. Valley, H. Kwon, G.C. Schatz and Y. Wang, Nat. Chem. 5, 840-845 (2013).

[8] B. E. Hardin, E. T. Hoke, P. B. Armstrong, J. H. Yum, P. Comte, T. Torres, J. M. J. Fréchet, Md. K. Nazeeruddin, M. Grätzel, M. D. McGehee, Nat. Photonics 3, 406 (2009).

[9] A.E. Nel, L. Mädler, D. Velegol, T. Xia, E.M.V. Hoek, P. Somasundaran, F. Klaessig, V. Castranova and M. Thompson, Nat. Mater. 8, 543 (2009)

[10] G. Magadur, J. S. Lauret, V. Alain-Rizzo, C. Voisin, Ph. Roussignol, E. Deleporte and J. A. Delaire, ChemPhysChem 9, 1250 (2008)

[11] J. P. Casey, S. M. Bachilo and R. B. Weisman, J. Mater. Chem. 18, 1510 (2008).

[12] J.K. Sprafke, S.D. Stranks, J.H. Warner, R.J. Nicholas and Harry L. Anderson Angew.Chem.Int.Ed.50, 2313-2316 (2011).

[13] F. Ernst, T. Heek, A. Setaro, R. Haag and S. Reich, Adv. Funct. Mater. 22; 3921-3926 (2012) 
[14] C. Roquelet, J.S. Lauret, V. Alain-Rizzo, C. Voisin, R. Fleurier, M. Delarue, D. Garrot, A. Loiseau, P. Roussignol, J. Delaire and E. Deleporte, ChemPhysChem 11, 1667 (2010).

[15] Y. Xu, L. Zhao, H. Bai, W. Hong, C. Li and G. Shi, J.A.C.S 131, 13490-13497 (2009).

[16] J.Geng and H.T. Jung, J.Phys.Chem.C 114, 8227-8234 (2010) .

[17] F. Vialla, G. Delport, Y. Chassagneux, P. Roussignol, J.S. Lauret and C. Voisin, Nanoscale 8, 2326 (2016).

[18] W. Orellana and J.D. Correa, J. Mat.Sci. 50, 898-905 (2015).

[19] http://www.nanointegris.com/en/puresheets .

[20] private communication with nanointegris.

[21] C.A. Silvera-Batista and K.J. Ziegler, Langmuir 27, 1137211380 (2011).

[22] KM Smith, JE Falk, Porphyrins and metalloporphyrins, Amsterdam: Elsevier, (1975).

[23] C. Roquelet, D. Garrot, J.S. Lauret, C. Voisin, V. AlainRizzo, P. Roussignol, J. Delaire and E. Deleporte, App. Phys. Lett. 97, 14 (2010).

[24] A.W. Adamson, Physical chemistry of surfaces, (1967)

[25] J.N. Weiss, FASEB J. 11, (1997) 835-841

[26] E.J.F. Carvalho and M.C. dos Santos, ACS Nano 4, 765-770 (2010).

[27] V.D.Pham, J. Lagoute, O.Mouhoub, F. Joucken, V. Repain, C. Chacon, A. Bellec, Y. Girard, and S. Rousset, ACS Nano 8, 9403-9409 (2014).

[28] W. Orellana, Chem.Phys.Lett 634, 47-52 (2015). 